\title{
EL ANÁLISIS DE LOS DIPUTADOS LATINOAMERICANOS EN EL CONTEXTO DE LOS ESTUDIOS SOBRE LA CLASE POLÍTICA. CARACTERÍSTICAS, OBJETIVOS Y ESTRATEGIAS DE INVESTIGACIÓN
}

\section{Manuel Alcántara Sáez e Iván Llamazares Valduvieco*}

\section{INTRODUCCIÓN}

El estudio de las élites parlamentarias en América Latina puede considerarse un apartado dentro del análisis global de los sistemas políticos de la región, un tema que ha sido explorado con anterioridad (Alcántara, 1989 y 1990). Asimismo, los estudios que se incluyen en este número se enmarcan dentro del análisis general de las élites, un análisis nunca abordado en términos continentales. En efecto, desde el clásico trabajo compilado por Lipset y Solari (1967) hasta el más reciente editado por Gunther (1992), los análisis han girado en torno a casos nacionales específicos (Bravo, 1986) o bien en torno a ámbitos sectoriales (élites militares, culturales, industriales, sindicales, campesinas, etc.), centrándose así en casos singulares a partir de los que se desarrollaba una comparación limitada. No existen, para dicha región, trabajos de la profundidad y el impacto del de Putnam (1973) para el caso de Gran Bretaña e Italia. De tal forma que nuestro desconocimiento de este terreno es sobresaliente.

Este abandono de los estudios generales acerca de las élites parlamentarias responde a motivos de diversa índole. En primer lugar, cabe reseñar el alto costo que supone, en términos económicos y humanos, la realización de investigaciones de este tipo. En segundo lugar, el hecho de que los Poderes Legislativos apenas desempeñaron un papel político relevante en la vida latinoamericana, fundamentalmente por la limitada estabilidad de la democracia en buen número de países de la región. Sin embargo, en la actualidad, la ola democratizadora ha venido acompañada de una generalizada conformación de parlamentos democráticos. Aunque el Poder Legislativo se ha visto históricamente marginado frente al Poder Ejecutivo (Lambert, 1973), la reivindicación de su papel es una característica actual en el debate politológico enmarcado tanto en la discusión propiciada por Linz et al. (1990) y Linz y Valenzuela (1994) que enfrenta a los vicios del presidencialismo con las virtudes del parlamentarismo, como por el papel del Parlamento en la etapa de consolidación democrática señalado por Santamaría (1994) y Cano y Porras (1994); para América Latina en su generalidad por Close (1995) y, más particularmente, para el caso de México por Lujambio (1993) y para el de Argentina por Molinelli (1991), por citar algunos de los más interesantes.

El estudio en este campo tiene, en primer lugar, la finalidad de describir a las élites parlamentarias latinoamericanas. Intenta así cubrir un vacío muy significativo. Pero al mismo tiempo, a partir de esta investigación se podrán realizar comparaciones entre la élite parlamentaria y los ciudadanos de cada sociedad.

\section{REPRESENTACIÓN, CLASE POLÍTICA Y PARLAMENTO. ALGUNAS CONSIDERACIONES PRELIMINARES}

El estudio de las élites políticas constituye uno de los ámbitos de atención preferente en la Ciencia Política. En esta línea de interés se inscriben los numerosísimos intentos de determinar, en una situación política determinada, las identidades, características y orientaciones de quienes gobiernan. Los trabajos aquí compilados, derivados de un ambicioso proyecto de investigación sobre los parlamentarios latinoamericanos, participan de un interés semejante en la exploración de las características e identidades de los políticos latinoamericanos. Ahora bien, el conocimiento de los individuos que poseen poder político no es estrictamente idéntico al de la clase política. A diferencia del concepto de élite política, el de clase política no alude especí-

* Universidad de Salamanca. 
ficamente a los individuos que dententan posiciones de especial poder e influencia, sino que se refiere al grupo, más extenso y también más fácilmente identificable, de los individuos que viven de la política y que poseen intereses y perspectivas peculiares como consecuencia de las posiciones que ocupan y de los papeles que desempeñan en la vida social (Borchert y Golsch, 1995: 612; Von Beyme,1995). Es dentro de este marco, y más específicamente, en referencia a los integrantes electos de los órganos legislativos latinoamericanos, en donde se inscriben las investigaciones que a continuación se presentan.

Ciertamente, uno de los componentes que define al campo de la política, y que constituye a su vez la propia naturaleza de las prácticas de los miembros de la clase política, es el de la representación. Sin representación, sin la expresión y recreación de las identidades y los intereses colectivos en la esfera de lo público, no cabe hablar de vida política (Schmitt, 1982: 208, 214; Bourdieu, 1990). Es por eso que una de las funciones primordiales de los políticos consiste en mostrar que existen aquellos grupos que son representados por los profesionales de la política. Esto es, esos grupos adquieren existencia política sólo en tanto en cuanto su presencia y demandas son simbolizadas y representadas en la esfera de lo político (Bourdieu, 1990). Esa labor de representación es característica de los integrantes de la clase política. A su vez, dicha representación remite a la pluralidad de identidades, valores e intereses que caracterizan a los grupos sociales. Como señaló Arendt (1993: 9), la misma existencia de la política está fundamentada en la pluralidad y la diferencia entre hombres que deben convivir juntos.

Del hecho de que la representación lo es de grupos con intereses, identidades y valores diferenciados se deduce que dicha práctica tiene un carácter necesariamente conflictivo. Ahora, al mismo tiempo, al menos en el marco cultural y político definido a partir de la experiencia de la Grecia antigua, la práctica de la representación implica, igualmente, una deliberación pública orientada a definir la acción de gobierno (Dunn, 1996: 29; Oakeshott, 1991).

Así pues, la vida política es indisoluble del concepto de representación. A su vez, esta representación lo es de una sociedad plural. Tiene un carácter necesariamente conflictivo y, al mismo tiempo, presupone la existencia de ciertas procesos de deliberación pública.

En las sociedades democráticas contemporáneas, las prácticas y procesos de representación política han adquirido contornos institucionales que, si bien incluyen muy importantes diferencias, como lo son sin duda las que separan a los regímenes presidenciales de los parlamentarios, adscriben a los órganos representativos de carácter legislativo una posición de especial relevancia. Esa posición se relaciona con las características centrales de la vida política. Cuando se piensa de forma específica en los individuos que se dedican de manera profesional a la política, los parlamentos, en tanto lugares privilegiados en los que tiene lugar la representación política, adquieren un papel aún más significativo. De ahí que, como han señalado Borchert y Golsch (1995: 613), el estudio de la clase política deba prestar atención prioritaria a los parlamentos y a sus inte- grantes. Esta importancia fundamental de la arena parlamentaria justifica nuestro interés prioritario, en el estudio de la clase política latinoamericana, en el análisis de los diputados de aquella región.

En este sentido, es preciso tener en cuenta que la clase política constituye un componente fundamental del sistema político de cada sociedad. Su análisis resulta imprescindible para una comprensión más adecuada de las estructuras sociopolíticas y de las dinámicas de conflicto. A su vez, este análisis implica establecer vínculos definidos entre el contexto político y social y las características de la clase política. En este terreno, resulta necesario recurrir a perspectivas teóricas que nos ayuden a definir el estudio de la clase política. Así, cuando se intenta explicar las características generales de la clase política a partir de su contexto de actuación, a nuestro juicio cabe hablar de cuatro grandes marcos explicativos (pluralista, estructuralista, culturalista, político-institucional). Los mismos predisponen al estudio de determinados factores, factores que son considerados los más relevantes en el estudio de este ámbito de análisis (Alford y Friedland,1985). Asimismo, estos marcos presuponen ciertas relaciones causales entre los distintos factores, o variables, presentes en una sociedad y en su sistema político. En el apartado siguiente, abordamos algunas de las características centrales de dichas alternativas teóricas.

Hay que precisar, en este sentido, que estas consideraciones iniciales acerca de los principales marcos teóricos remiten especialmente a las explicaciones acerca de las características fundamentales de la clase política. En ese sentido, enfatizan los elementos contextuales que condicionan los rasgos fundamentales de la clase política. Este tipo de explicación concierne, fundamentalmente, a la representatividad de la clase política (sus condiciones sociales y políticas de partida), al reclutamiento de ésta y a su profesionalización ${ }^{1}$. Esta explicación es necesaria en la medida en que otros elementos fundamentales para el análisis de la clase política (de manera especial, aquellos que se refieren a la acción de sus integrantes), requerirían la inclusión de consideraciones adicionales ${ }^{2}$.

\section{ALTERNATIVAS TEÓRICAS FUNDAMENTALES EN EL ESTUDIO DE LA CLASE POLÍTICA}

\section{A. Algunas consideraciones teóricas acerca del análisis de la acción y las orientaciones de la clase política}

A la hora de describir las principales corrientes de análisis sobre la acción y orientaciones de la élite política, cabría distinguir, extendiendo y simplificando las clasificaciones elaboradas por Searing sobre los estudios en torno a los roles de los políticos, entre tres grandes tipos de estilos

1. Sobre estos factores, ver Borchert y Golz (1995)

2. Sobre las distintas aproximaciones a este tipo de análisis, ver Searing (1994) y Evans (1995). 
de análisis ${ }^{3}$. En primer lugar estarían las visiones de tipo estructural, utilizando este término en el sentido más amplio posible. Las mismas sitúan como condicionantes fundamentales de la orientación de las élites políticas su origen, sus vínculos sociales y su acceso a canales de poder e influencia. Es importante reseñar aquí que dichas visiones estructurales pueden tener tanto un carácter economicista como un sesgo culturalista. Lo fundamental es que, desde este punto de vista, la autonomía del campo político es extremadamente limitada. Consiguientemente desde esta perspectiva, las acciones y estrategias de los actores políticos dependerán fundamentalmente de las características culturales, sociales, económicas o institucionales en las que están inmersos los actores políticos ${ }^{4}$.

En segundo lugar, se inscribirían los estudios denominados interaccionistas. Los mismos estipulan que las orientaciones y la acción de las élites políticas surgen de los procesos de interacción que tienen lugar dentro de la élite política. En este marco de análisis, sería el contexto (si bien de un carácter más limitado en este caso, en la medida en que el mismo quedaría limitado a las interacciones entre los políticos), el que condicionaría decisivamente las acciones de estos últimos. A su vez, esas interacciones serían entendidas desde una perspectiva eminentemente psicológica ${ }^{5}$.

Finalmente, en un tercer plano cabría situar las visiones que han sido calificadas como de motivacionistas. Las mismas insisten en la importancia conjunta de, en primer lugar, las orientaciones que los políticos tenían antes de ocupar sus cargos y, en segundo lugar, las motivaciones y propósitos que los mismos desarrollan en el curso de sus actividades (Searing, 1994: 10-11). En este contexto, la racionalidad de los políticos es el determinante básico de su propia creatividad. Debido a ello, el estudio de los contextos en los que se mueven los políticos, y de los rasgos básicos de las interacciones entre aquellos, no aseguran una correcta comprensión de las acciones de estos últimos. En este tipo de análisis, las motivaciones y la racionalidad de los políticos pasan a constituir un ingrediente fundamental del análisis de la acción política. De ahí que para entender la acción política resulte imprescindible entender como los propios políticos perciben e interpretan su propia posición, sus finalidades y los medios a su alcance. En este sentido, la racionalidad de la acción política condiciona la creatividad de ésta, el hecho de que ésta no pueda ser anticipada por un análisis de tipo estructural del tipo que sea ${ }^{6}$.

A nuestro juicio, esta última orientación teórica es extraordinariamente sugerente, y puede orientar el desarrollo de ambiciosas investigaciones sobre las motivaciones y los objetivos de la acción de los integrantes de la clase política. En ese sentido, algunas de las preguntas que subyacen a esta investigación están directamente relacionadas con la preocupación por la forma en que los políticos se ven a sí mismos y a sus respectivos contextos políticos e institucionales.

Al mismo tiempo, a fin de profundizar en el estudio de estas motivaciones, resulta imprescindible determinar, desde una perspectiva comparativa, cuales son los rasgos básicos de la clase política, de sus patrones de reclutamiento y de su actividad cotidiana. En este sentido, el análisis de los determinantes básicos, externos si se quiere, de la composición y rasgos de la clase política, constituye un ingrediente fundamental del estudio de esta última. Las investigaciones que se incluyen aquí, basadas en las encuestas realizadas a los diputados latinoamericanos, dan precisamente una idea muy definida de las potencialidades del análisis de este campo temático. En ese sentido, proporcionan información básica sobre los elementos estructurales antes mencionados. Ahora bien, en este terreno resulta necesario determinar cuales son las alternativas teóricas en juego. Sobre estos elementos trata precisamente el siguiente apartado.

\section{B. El estudio de los patrones de representación y reclutamiento de la clase política latinoamericana}

Un primer marco explicativo en el estudio de las características de la clase política sería aquel que subraya el peso de los factores culturales en la determinación de las características (y orientaciones) de la clase política. En este terreno, cabría distinguir dos vertientes de análisis. Una primera consistiría en el análisis estrictamente culturalista. En el estudio de Latinoamérica, esta vertiente ha subrayado las tradiciones ideológicas y culturales específicas de esta región. Este análisis ha girado en torno al pretendido vigor de los valores culturales heredados del mundo hispánico colonial. De ahí que los autores que han analizado la política de estas sociedades desde la clave culturalista, hayan recurrido a los elementos corporativistas y tomistas como ingredientes fundamentales en la constitución de las sociedades latinoamericanas. En este terreno se incluyen, por ejemplo, los estudios de Wiarda (1981).

Una versión más compleja del estudio de los valores culturales de las sociedades latinomericanas consistiría en el análisis centrado en los procesos de modernización, análisis realizado desde una perspectiva pluralista. Dicha perspectiva, especialmente vigorosa en la ciencia política norteamericana posterior a la II Guerra Mundial, se caracterizaría por subrayar la importancia explicativa central de los valores culturales que predominan en la sociedad. En este sentido, esta orientación teórica tiene un fuerte componente culturalista (Lipset, 1967). En segundo lugar, esta perspectiva subrayaría la vinculación especial de los distintos fragmentos de la clase política con los distintos grupos sociales y culturales presentes dentro de cada sociedad. En ambos sentidos, cabría pensar en la clase política como expresión de

3. Sobre estos puntos, ver Searing (1994: 1-24).

4. Entre los estudios clásicos que representan este tipo de visión podríamos citar el de Wahlke, Eulau, Buchanan y Ferguson (1962).

5. Para un ejemplo de este tipo de perspectiva, ver Cain, Ferejohn y Fiorina (1979).

6. Para este tipo de análisis, ver especialmente Aberbach, Putnam y Rockman (1981). Ver igualmente Payne, Woshinsky, Veblen, Coogan y Bigles (1990). Este tipo de perspectiva tiene significativos puntos de contacto con la concepción de la política de Hannah Arendt. Es igualmente consistente con la aproximación interpretativa al estudio de la acción económica Piore (1995: 119-139). 
ciertas características generales de la sociedad. Esta explicación tendría, por tanto, un carácter marcadamente "sociocéntrico". Finalmente, la aproximación pluralista subrayaría la importancia de los procesos de desarrollo social y económico en la modificación de las características de las élites políticas. Dichos procesos llevarían necesariamente a la movilización política de nuevos sectores, a la modernización de las actitudes y valores de las élites.

Para evaluar el valor expliativo de estas perspectivas sería necesario establecer, en primer lugar, en qué medida estamos, en la región latinoamericana, en presencia de situaciones de alto consenso cultural, al menos por lo que se refiere a las orientaciones de tipo político. Un grado intenso de diferencias ideológicas en la clase parlamentaria cuestionaría las interpretaciones más simples realizadas desde una aproximación culturalista. Asimismo, sería necesario establecer en qué medida los trasfondos sociales y los patrones de reclutamiento de la clase política señalan la presencia de sociedades altamente corporativas y exitosamente jerarquizadas. En este sentido, sería necesario determinar en qué medida el consenso cultural y político es mayor, como parecería sugerir una interpretación culturalista, en las sociedades que han experimentado menos transformaciones sociales y que permanecen por tanto más cercanas al mundo cultural colonial e hispánico. Por otra parte, de ser ciertas las presunciones de estos enfoques culturalistas, en las sociedades latinoamericanas más modernizadas los integrantes de la clase política mostrarían no sólo orientaciones más liberales en el terreno político, sino también actitudes menos intervencionistas y estatistas en el orden económico.

Un segundo tipo de aproximación al estudio de la clase política consistiría en el énfasis en el peso de los elementos de estructura social. Desde esta perspectiva, la composición de clases de cada sociedad, la fuerza social de los grandes propietarios agrícolas, el tamaño de la clase obrera y las relaciones económicas establecidas entre las sociedades latinoamericanas y los centros económicos internacionales, contribuyen a definir el perfil social y político de las sociedades latinoamericanas. Además, estas características específicas de las sociedades latinoamericanas habrían condicionado la intensidad y polarización de los conflictos políticos en cada país, así como las características y estabilidad de los regímenes políticos. Consiguientemente, las características de la clase política se relacionarían de manera muy precisa con los rasgos fundamentales de la estructura social. Cabría hablar así, por ejemplo, de sociedades en las que estaría presente una movilización muy significativa de los sectores campesinos pobres y asalariados, con una tradición de sistemas políticos oligárquicos y/o excluyentes y con un alto nivel de polarización política. Dichas sociedades se caracterizarían por la vulnerabilidad notable de la política democrática. A su vez, esta vulnerabilidad habría llevado a una deficiente institucionalización de la clase política de esas sociedades. Esta última tendría, bien rasgos estrictamente oligárquicos (en los sistemas políticos más cerrados de la región), bien rasgos altamente polarizados y disímiles por lo que se refiere a la clase política (en lo que se refiere a su trasfondo social, reclutamiento político, motivaciones y orientaciones ideológicas de partida). En estos casos se podría hablar, igualmente, de una deficiente institucionalización de la clase política. Este hecho podría ser rastreado en el análisis de los parlamentos de cada país. El mismo remitiría tanto al consenso sociopolítico como al grado de profesionalización de la clase política y a su diferenciación respecto a otros grupos sociales. Simétricamente, en aquellas sociedades de la región en las que se hubiese producido un desarrollo socioeconómico significativo, el desarrollo y la complejización de la sociedad, los elementos de polarización social e intensidad de conflictos políticos incidirían en una mayor profesionalización de la clase política, así como en un nivel menos acentuado de división entre los integrantes de la clase política.

Una última aproximación a la clase política latinoamericana consistiría en la indagación preferencial en los componentes del sistema político-institucional de estas sociedades. En este terreno, dos son los ingredientes que parecerían fundamentales. En primer lugar, las características del sistema de partidos de cada sociedad. En este punto, son variables fundamentales las que se refieren a la institucionalización de los partidos políticos, al número de éstos en cada sistema político, a la polarización ideológica, a la existencia de retos insurgentes o reactivos en el marco político, y a los vínculos de representación entre fuerzas políticas y grupos sociales. Además, esta aproximación subrayaría la importancia de las dinámicas de conflicto de partidos experimentadas por cada sociedad. En este terreno, esta aproximación no es incompatible con el desarrollo de análisis sensibles a los factores socioeconómicos y culturales de cada sociedad.

Un segundo factor crítico de corte institucional, relacionado igualmente con los patrones de representación y reclutamiento de los actores políticos, consistiría en las características institucionales de cada régimen político. Aquí resultan críticos factores como el presidencialismo, las características federales o centralizadas del sistema político, el sistema electoral, las normas especificas que regulan las relaciones entre las cámaras y entre las cámaras y el ejecutivo, etc. Esta vertiente de análisis se inserta obviamente en los estudios de corte institucionalista y, de manera especial, en los análisis enmarcables en el institucionalismo de tipo histórico. En este terreno, las formas históricas específicas en que se han relacionado normas institucionales, prácticas políticas y conflictos partidarios resultan claves a la hora de explicar las características y orientaciones de los diputados latinoamericanos.

\section{PROBLEMAS CONCEPTUALES Y CAMPOS DE ANÁLISIS FUNDAMENTALES}

La presente investigación constituye, por tanto, un punto de partida importante que persigue ofrecer resultados empíricos que tengan a su vez implicaciones teóricas. En las páginas que siguen, repasamos los campos de análi- 
sis fundamentales en los que se sitúan los datos obtenidos mediante la realización de extensas y estructuradas encuestas a los diputados latinoamericanos. Estos campos se relacionan a su vez con ámbitos específicos de problemas teóricos, ámbitos que se han tenido en cuenta en la confección de esta encuesta.

Un primer campo se refiere específicamente a la definición y caracterización de un segmento de la clase política latinoamericana: los diputados. En este terreno, resulta de especial interés la determinación de las eventuales peculiaridades de los diputados latinoamericanos y de su relación con el concepto de "clase política" recientemente avalado por Von Beyme (1995). Este análisis ha de fundamentarse en un estudio descriptivo y teóricamente articulado de las características y experiencias políticas y profesionales de los diputados latinoamericanos.

Un segundo campo de análisis se refiere a la forma en que las estructuras sociales y dinámicas políticas de cada país han condicionado las características y orientaciones de la élite política. En este sentido, este tipo de análisis pretende dilucidar el grado en el que las variables sociales y políticas explican las características y orientaciones de los diputados latinoamericanos. Estas últimas aparecerían como las variables dependientes de este tipo de análisis. Es importante tener en cuenta que los factores explicativos considerados aquí tienen un carácter tanto social como estrictamente político. El estudio de su importancia requiere un análisis cuidadoso que tenga en cuenta tanto las características e historias personales de los diputados como otros factores contextuales que no aparecen de manera explícita en los datos ofrecidos por la encuesta. Pensemos, en este punto, en la evolución de los conflictos políticos en la zona (prohibición de determinadas fuerzas políticas, polarización ideológica, cambios institucionales, política internacional), así como en las características de la estructura social de cada una de estas sociedades .

Por lo tanto, estos análisis remiten a las relaciones que existen entre los niveles macro y micro del análisis político. $\mathrm{Al}$ informarnos sobre las características sociales y económicas de los diputados, sus experiencias políticas y asociativas y sus orientaciones ideológicas, esta encuesta brinda información de primera utilidad para juzgar, en primer lugar, en qué formas las historias singulares de los países latinoamericanos han condicionado las trayectorias vitales, las experiencias políticas y las actitudes ideológicas y culturales de los parlamentarios de aquellos países. En este punto, la comparación entre los países latinoamericanos abre nuevas posibilidades a la hora de determinar los condicionantes de las actitudes de los parlamentarios hacia los regímenes políticos, hacia determinadas políticas públicas, y hacia la vida política en general.

Un tercer campo de análisis se refiere a los vínculos entre las actitudes y estilos políticos reflejados por estas encuestas y las dinámicas del sistema político. En este sentido, esta investigación sigue las líneas básicas articu- ladas por Putnam (1973). Como se recordará, dicho estudio indagaba en los factores que determinaban las creencias fundamentales de los políticos (por lo que se refería a su estilo político, sus predisposiciones cognitivas y sus valores y normas). Al mismo tiempo, dicho estudio pretendía establecer las formas en que tales creencias afectaban la acción política y, de este modo, a los mismos regímenes políticos. Pues bien, este estudio continúa esa misma línea de desarrollo en un proyecto si cabe más ambicioso, en la medida en que abarca núcleos temáticos más amplios que los tocados por el pionero estudio de Putnam y en que aborda un número mucho más alto de sociedades. En este sentido, el presente estudio abre mayores posibilidades a la utilización de técnicas cuantitativas en la medida en que amplía tanto el número de diputados entrevistados como el número de sociedades estudiadas. Estas características deben permitir una evaluación rica y sistemática de los distintos factores que inciden en la formación de las orientaciones políticas de los parlamentarios.

El interés de este tipo de análisis se percibe de manera más clara al abordar las cuestiones específicas con las que se relaciona. Así, esta encuesta permite abordar problemas tan importantes como el de la polarización ideológica y el de las actitudes hacia la democracia (o, más específicamente, hacia los distintos componentes de la misma). En el primer punto, a partir de esta encuesta es posible comparar los elementos de polarización en los distintos sistemas de partidos de la región, la articulación de las diferencias ideológicas de tipo espacial (izquierda-derecha) a través de los partidos políticos y, en último término, la validez de la misma distinción izquierda-derecha en Latinoamérica (Alcántara, 1991 y 1996).

En este terreno, la encuesta realizada a los diputados latinoamericanos permite explorar la solidez de las convicciones democráticas entre los mismos, así como estable, a través de análisis individuales y comparativos, los determinantes fundamentales de dichas convicciones. En este sentido, la Tabla 1 revela tanto la fuerza de las manifestaciones de fe democráticas (en el total de los diputados latinoamericanos, un $98 \%$ está muy de acuerdo o de acuerdo con la idea de que la democracia es el mejor de los sistemas políticos) como la existencia de algunas especificidades nacionales dignas de ser reseñadas. En este sentido destacan, por el alto porcentaje de quienes se muestran muy de acuerdo con dicha idea, los casos de Costa Rica, Nicaragua, Argentina, Paraguay, Uruguay, Brasil y Panamá. El porcentaje de quienes se muestran en desacuerdo o muy en desacuerdo con dicha idea es o muy bajo o inexistente en todos los países, si bien hay bolsas un poco más significativas de reticencia en los casos de Guatemala (10\%) y Perú (6\%). Resulta interesante reseñar que precisamente en Honduras, El Salvador y Guatemala el porcentaje de muy alto acuerdo con la idea de que la democracia es el mejor sistema político posible está entre los menores de la región. 
TABLA 1

Grado de acuerdo, por países, con la idea de que la democracia es el mejor sistema político posible (los porcentajes se suman horizontalmente).

\begin{tabular}{|c|c|c|c|c|c|c|}
\hline & $\begin{array}{l}\text { Muy de } \\
\text { acuerdo }\end{array}$ & $\begin{array}{c}\text { De } \\
\text { acuerdo }\end{array}$ & $\begin{array}{c}\text { En } \\
\text { desacuerdo }\end{array}$ & $\begin{array}{c}\text { Muy en } \\
\text { desacuerdo }\end{array}$ & NC & $n$ \\
\hline COSTA RICA & 90 & 10 & & & & 52 \\
\hline EL SALVADOR & 54 & 44 & 2 & & & 46 \\
\hline HONDURAS & 63 & 37 & & & & 67 \\
\hline NICARAGUA & 90 & 7 & 3 & & & 41 \\
\hline ARGENTINA & 98 & 2 & & & & 58 \\
\hline COLOMBIA & 81 & 14 & 2 & 3 & & 63 \\
\hline VENEZUELA & 89 & 7 & 4 & & & 69 \\
\hline REP. DOMINICANA & 80 & 18 & 2 & & & 62 \\
\hline MÉXICO & 84 & 12 & 3 & 1 & & 123 \\
\hline CHILE & 80 & 18 & 1 & 1 & & 94 \\
\hline GUATEMALA & 65 & 25 & 5 & 5 & & 20 \\
\hline PERÚ & 79 & 15 & 5 & 1 & & 87 \\
\hline PANAMÁ & 92 & 6 & 2 & & & 71 \\
\hline BRASIL & 92 & 8 & & & & 61 \\
\hline URUGUAY & 93 & 7 & & & & 73 \\
\hline BOLIVIA & 83 & 17 & & & & 46 \\
\hline PARAGUAY & 92 & 6 & 2 & & & 47 \\
\hline ECUADOR & 89 & 9 & 2 & & & 71 \\
\hline TOTAL & 82 & 16 & 2 & & & 1.135 \\
\hline
\end{tabular}

Además, esta encuesta ofrece información precisa acerca de lo que los diputados piensan sobre los distintos componentes de los regímenes democráticos (elecciones, partidos políticos, libertades políticas, formas institucionales, participación ciudadana, etc.). Dicho análisis es fundamental tanto si se confronta el problema clásico de entender los factores de fuerza o debilidad de la democracia en la región, como si se evalúan las potencialidades, en cada país de la zona, de las orientaciones de tipo antipartido, canalizadas por líderes como Fujimori. En último término, esta encuesta permite analizar las orientaciones de los diputados hacia las distintas formas institucionales, $y$, por tanto, la viabilidad de las reformas de los regímenes presidencialistas. Esto es, el estudio de las distintas cuestiones implicadas en el análisis de la democracia resulta empíricamente factible a partir de los resultados proporcionados por esta encuesta.

Igualmente dentro de este segundo campo se situaría el estudio de los factores que inciden sobre las actitudes de los diputados hacia ciertos problemas fundamentales de la política pública de las sociedades latinoamericanas. En este terreno se sitúan todas las cuestiones relacionadas con las reformas económicas experimentadas por los países latinoamericanos. Estas cuestiones tienen implicaciones tanto económicas como políticas. De una manera u otra, los gobiernos latinoamericanos han afrontado procesos de reforma del Estado y de liberalización económica de especial significación. Dichos procesos se han realizado a menudo en situaciones políticas en las que el Presidente carecía del apoyo necesario por parte del Congreso. En este marco político no ha resultado infrecuente que los Presidentes actuasen utilizando vías de urgencia y eludiesen así el respaldo del Congreso para medidas de difícil tramitación parlamentaria. Esta tendencia al "ejecutivismo" puede tener implicaciones para la continuidad y viabilidad de las reformas económicas (Bresser Pereira, Maravall y Przeworski, 1993). Además, esta tendencia tiene efectos directos sobre rasgos fundamentales de la vida democráti$\mathrm{ca}$, como se percibe en los debates acerca de la pertinencia de etiquetar de manera específica a algunos regímenes democráticos de la región (O'Donnell, 1996). Estas cuestiones afectan igualmente a la gobernabilidad de sistemas políticos caracterizados por trayectorias especialmente inestables (Alcántara, 1994).

A este respecto, la Tabla 2 refleja precisamente la gravedad adjudicada por los diputados latinoamericanos a la crisis económica experimentada por sus respectivos países en el momento de realización de la entrevista. Resulta muy revelador inspeccionar el grado de gravedad de la crisis señalado por los diputados de cada país, con Bolivia, Ecuador, Nicaragua y Venezuela como los cuatro países cuyos diputados veían con mayor preocupación la crisis económica. En el lado opuesto del abanico se sitúa el caso chileno y también, aunque con una situación menos cómoda que la chilena, Uruguay, Argentina y Perú. 


\section{TABLA 2}

Evaluación media de la gravedad de la crisis económica por parte de los diputados de cada país (dónde 1 indica mínima gravedad y 5 máxima gravedad).

\begin{tabular}{||l|c|c|c||}
\hline & Media & Desv. típ. & $n$ \\
\hline BOLIVIA & 4.9 & 0.3 & 47 \\
\hline ECUADOR & 4.7 & 0.7 & 71 \\
\hline NICARAGUA & 4.6 & 0.8 & 41 \\
\hline VENEZUELA & 4.4 & 0.8 & 69 \\
\hline HONDURAS & 4.2 & 1.1 & 67 \\
\hline REP. DOMIN. & 4 & 1.1 & 61 \\
\hline MÉXICO & 4 & 1.2 & 123 \\
\hline PARAGUAY & 4 & 0.9 & 47 \\
\hline EL SALVADOR & 3.9 & 1.1 & 46 \\
\hline PANAMÁ & 3.7 & 1.2 & 54 \\
\hline GUATEMALA & 3.6 & 1.1 & 19 \\
\hline COLOMBIA & 3.6 & 1.1 & 63 \\
\hline COSTA RICA & 3.5 & 0.9 & 51 \\
\hline BRASIL & 3.4 & 1.4 & 59 \\
\hline PERÚ & 3.3 & 1.2 & 87 \\
\hline ARGENTINA & 3.2 & 1.2 & 56 \\
\hline URUGUAY & 3.1 & 1.2 & 73 \\
\hline CHILE & 2.4 & 1.3 & 94 \\
\hline TOTAL & 3.7 & 1.2 & 1128 \\
\hline \hline
\end{tabular}

En último término, el estudio de los campos y factores reseñados más arriba se orienta a una definición más completa y más precisa de los distintos componentes de los sistemas políticos de cada sociedad latinoamericana. Esta definición se relaciona con distintas hipótesis acerca de la relevancia de unos y otros factores en la explicación de la vida política. A su vez, estas hipótesis se vinculan a expectativas acerca de la importancia causal de determinados factores y variables. Estas expectivas tienen importantes implicaciones teóricas. Las mismas remiten a marcos teóricos diferenciados en el análisis de la vida política. En las líneas que siguen, no pretendemos hacer un repaso exhaustivo de los marcos teóricos en vigor, ni juzgar la propiedad definitiva de los mismos, sino simplemente reseñar el interés de estas investigaciones para evaluar la validez de distintos marcos teóricos. A nuestro juicio, el análisis estructural, las perspectivas culturalistas y el institucionalismo de distintos tipos son algunas de las perspectivas más relevantes en este terreno.

Así, los problemas mencionados arriba se relacionan directamente con los campos de problemas definidos por los análisis de tipo estructural. Los estudios sobre los fac- tores sociales y económicos que condicionan la naturaleza de las élites políticas latinoamericanas tienen mucho que ver con la expectativa de que dichos factores juegan un papel muy relevante en la dinámica política de aquellas sociedades. Esta expectativa es compatible con el reconocimiento de la importancia de la vida política y partidista, pero resalta las constricciones que el nivel de desarrollo y la estructura de clases de cada sociedad imponen a la vida política (Rueschemeyer, Huber Stephens y Stephens, 1992). En este sentido, resulta interesante comprobar, en el análisis de los diputados, si los factores de este tipo han condicionado las características no sólo de los diputados, sino de los partidos y los sistemas de partidos de cada sociedad. Además, y en relación con los argumentos de esos mismos autores, tiene interés especial delimitar la pujanza de la sociedad civil en cada sociedad y su vinculación con la socialización política de los diputados.

En este terreno, la encuesta permite analizar los vínculos que existen entre los procesos de desarrollo económico y cambio social de cada sociedad y profesionalización de la élite parlamentaria. Los análisis de este tipo permiten comprobar la validez que tienen para las sociedades centroamericanas estudios previos acerca de la relación entre el desarrollo socioeconómico y los procesos de diferenciación y profesionalización de la élite política en las sociedades contemporáneas (Best, 1995; Matthews, 1985; King, 1981; Eliassen, 1995; Ruostetsaari, 1995), así como establecer contrastes significativos entre los países analizados aquí.

Como revela la tabla 3 , el porcentaje de diputados que señala dedicarse de manera exclusiva a la actividad política ronda como media el $45 \%$. Resulta interesante el hecho de que los más altos porcentajes de dedicación exclusiva a la política se den en países centroamericanos, como Honduras, El Salvador, Guatemala y Panamá. Por contra, en países como Brasil, Bolivia, la República Dominicana, México, Argentina y Chile dichos porcentajes son mucho más limitados. Por otra parte, si bien esta tabla apunta una mayor dedicación exclusiva a la política en los países centroamericanos, resultaría necesario, a fin de evaluar el grado de profesionalización de la clase política, establecer cuales son las ocupaciones y cualificaciones de los diputados en cada país y para cada partido político.

La investigación de las élites parlamentarias proporciona información fundamental también sobre los factores de tipo valorativo en la vida política de la región. En este sentido, permite analizar, para este grupo específico, los condicionantes y efectos de factores que han sido considerados críticos por determinadas corrientes teóricas. En las mismas se inscriben los análisis tradicionales de la cultura política, así como toda la corriente que ha subrayado las características culturales idiosincráticas del área latinoamericana (Wiarda, 1981). A partir de esta investigación es posible sopesar el peso en las élites políticas de los distintos países y partidos latinoamericanos de orientaciones ideológicas (como el corporativismo, el autoritarismo, el estatismo, 
TABLA 3

Dedicación exclusiva a la política por países (en porcentajes).

\begin{tabular}{||l|c|c|c||}
\hline & Si & No & $n$ \\
\hline COSTA RICA & 52 & 48 & 50 \\
\hline EL SALVADOR & 59 & 41 & 44 \\
\hline HONDURAS & 88 & 12 & 67 \\
\hline NICARAGUA & 50 & 50 & 40 \\
\hline ARGENTINA & 30 & 70 & 57 \\
\hline COLOMBIA & 44 & 56 & 63 \\
\hline VENEZUELA & 41 & 59 & 69 \\
\hline REP. DOMIN. & 22 & 78 & 62 \\
\hline MÉXICO & 33 & 67 & 123 \\
\hline CHILE & 22 & 78 & 94 \\
\hline GUATEMALA & 65 & 35 & 20 \\
\hline PERÚ & 35 & 65 & 86 \\
\hline PANAMÁ & 58 & 42 & 53 \\
\hline BRASIL & 32 & 68 & 57 \\
\hline URUGUAY & 44 & 56 & 73 \\
\hline BOLIVIA & 21 & 79 & 47 \\
\hline PARAGUAY & 43 & 57 & 47 \\
\hline ECUADOR & 50 & 50 & 70 \\
\hline TOTAL & 45 & 55 & 1122 \\
\hline
\end{tabular}

etc.), así como los condicionantes individuales y estructurales de las actitudes generales ideológicas de los diputados latinoamericanos.

Finalmente, esta investigación puede proporcionar información de especial interés a los estudios de tipo institucional, que tanta importancia han adquirido en años recientes. La información proporcionada por estas encuestas debe permitir analizar las formas en las que los marcos institucionales de los países latinoamericanos (por lo que se refiere a las relaciones entre el parlamento y la presidencia, y a la propia vida institucional de las cámaras bajas de aquella región) se relacionan con la vida política y la lucha interpartidista de los países analizados. En los últimos años, numerosos estudios han señalado la existencia de vinculaciones directas entre marcos institucionales y orientaciones y vida política (Thelen y Steinmo, 1992; y Rohrschneider, 1994). Además, son ya numerosos los estudios que han abordado las implicaciones de los marcos institucionales presidenciales para las perspectivas de la democracia en Latinoamérica (Linz y Valenzuela, 1994). Esta investigación proporciona información detallada sobre cuestiones de trascendencia referidas a estos problemas, cuestiones que incluyen las relaciones interpartidarias en el ámbito parlamentario, el papel del parlamento en la producción legislativa de aquellas sociedades, o las relaciones entre este último y el poder ejecutivo. En este sentido, el presente estudio puede brindar información utilísima a la hora de evaluar la importancia de los distintos marcos jurídico-políticos sobre la vida parlamentaria y, por derivación, sobre la vida política en Latinoamérica.

En último término, si bien los estudios que se recogen en este número no responden a una perspectiva teórica homogénea, los mismos pretenden ofrecer elementos de juicio de primera importancia para evaluar la validez de unas y otras expectativas. Ello resulta más patente cuando se presta atención especial a la forma específica en que estos marcos teóricos se relacionan con campos de interés e investigación como los que se definen a continuación.

\section{PRINCIPALES VARIABLES EN LA INVESTIGACIÓN DE LAS ÉLITES}

Una investigación de las características como la presente, tiene por objeto el estudio de los valores, actitudes políticas, formas de adscripción partidista y elementos biográficos de la élite parlamentaria de los diferentes países de América Latina. Si bien, en una aproximación inicial, la investigación va a seguir el método del estudio de casos a nivel nacional (circunstancia que por sí misma ha generado un importante flujo de información para la realización de estudios monográficos nacionales), el fin último de la misma reivindica el método comparado. De esta forma, de acuerdo con las ventajas de dicho método establecidas por Lijphart (1971), Smelser (1976) y Collier (1991) entre otros, y con la opción metodológica expresamente defendida por Alcántara (1993), la comparación de los distintos casos nacionales se considera como un método de trabajo suficientemente fuerte y capaz de producir resultados idóneos.

Por consiguiente, los datos que se han obtenido del instrumento básico de este estudio que representa un cuestionario, se están utilizando para realizar tres tipos de análisis: estudios de caso, estudios comparados y análisis estadísticos. En el estudio de caso, de diseño más intensivo se pueden analizar un mayor número de variables relativas a cada caso. El resultado de este tipo de análisis no será exclusivamente descriptivo en la medida en que su realización se oriente a confirmar o refutar expectativas de tipo teórico. Los estudio de casos "cruciales" son un buen ejemplo de como un estudio de este tipo puede servir para reforzar o cuestionar marcos teóricos. Esto es, los estudios de este tipo pueden servir para establecer inferencias cuyo valor trascienda al caso analizado. En todo caso, el diseño de esta investigación ha sido guiado por el interés de realizar análisis comparativos, análisis que permitan extraer conclusiones generales acerca de las diferencias y similitudes entre las características y orientaciones de las élites parlamentarias. Estos análisis permitirán además evaluar las causas de dichas orientaciones y características. En último término, esta investigación permite aplicar técnicas de tipo 
estadístico. Las mismas podrán enmarcarse igualmente en estudios cuyo diseño global sea de tipo comparativo. Esta última parte más analítica de la explotación de los datos y de prueba de hipótesis se desarrollará mediante la utilización de distintas técnicas estadísticas que variarán de acuerdo con diversos factores, principalmente el carácter de las variables dependientes e independientes. El hecho de que las variables que se consideran en el análisis sean nominales, ordinales o intervales conlleva la utilización de técnicas distintas. Asimismo, el tipo de técnica escogida dependerá del tipo de hipótesis que se estén probando.

A la hora de diseñar el cuestionario, se abandonó la senda iniciada por Putnam (1973) al optar por uno de estructura cerrada en contra del abierto utilizado por dicho autor. Por otra parte, se comparte la búsqueda de los denominados "ideales operativos" o conjunto de valores que guían la actividad de los políticos tanto de manera retórica como simbólica. Las tres facetas que se pretenden cubrir son las siguientes: el análisis de la democracia, el análisis del sistema político del país en cuestión y el análisis de la política como profesión. Complementariamente se abre un campo de datos biográficos personales que da cabida a las principales variables independientes de la investigación.

1) El análisis de la democracia se efectúa a través de su definición, la evaluación de sus ventajas, de los aspectos cualitativos ligados a la consolidación y al riesgo de involución; del papel del sistema electoral, de los partidos políticos y del grado de movilización popular. Este análisis permitirá configurar un esquema global en el que se dieran cabida al encasillamiento de los diputados según un modelo de democracia que pudieran suponer cinco tipologías derivadas de la literatura más clásica sobre los estudios acerca de la democracia (Sartori, 1988; Cotarelo, 1990; Linz, 1992; Requejo, 1990) y que serían: democracia electoral, procedimental, participativa, económica y personal.

La democracia electoral vendría ligada por la conjunción de las siguientes características: elegir a las autoridades como principal ventaja de la democracia, las elecciones como el mejor método, la consolidación democrática sinónimo de plena confianza en las elecciones, facilitar al elector confeccionar las listas y la participación ciudadana limitada a las elecciones. En cuanto a la democracia procedimental recogería las ideas de solución de problemas de forma pacífica, en lo atinente para la consolidación - consenso sobre instituciones básicas, descentralización, constitucionalidad, imperio de la ley-, con respecto al sistema electoral — garantizar la representación, conformar mayorías, facilitar el desarrollo de partidos coherentes, renovar líderes políticos-, y legalización de los partidos políticos. En tercer lugar, la democracia participativa estaría constituída por posiciones manifiestas en torno a poder participar en las decisiones, a dar facilidades a las minorías, al grado ideal de militancia partidista, al modo de la misma, a la actividad ideal de los movimientos sociales y al futuro de los mismos. Con respecto a la democracia económica se basaría en integrar visiones que defienden que con la democracia la economía crece más, disminuye la pobreza y se distribuyen los ingresos, que la consolidación necesita acuerdos económicos y que la quiebra de la democracia se debió a la crisis económica. Finalmente, la democracia personal se refiere a la existencia de oportunidades de desarrollo personal, de mejora de la convivencia, de la libertad de las personas y de los derechos humanos.

La adscripción a estos modelos se correlacionará con la vinculación partidista con el fin de comprobar si la pertenencia a un partido comporta concepciones diferentes de la democracia. En la vinculación partidista, mediante el grado de identificación con el partido, se pretende corroborar las hipótesis elaboradas por Westholm y Niemi (1992) relativas a si la identificación partidista se conforma antes que la mayoría de las otras actitudes políticas y se encuentra fuertemente influenciada por los puntos de vista del padre; si permanece estable a lo largo de la vida; y si afecta fuertemente a otras actitudes políticas, sirviendo como un punto de anclaje para compromisos políticos y el establecimiento de una agenda política determinada. Así mismo se buscará la correlación existente con las variables que perfilan la situación socioeconómica del diputado, con la de sus valores que se señalan más adelante y con la autoubicación en la escala izquierda-derecha.

2) El análisis del sistema político del país, en primer lugar, sobre la base del grado de satisfacción con el mismo medido por su estabilidad, la confianza en las elecciones y el grado de amenazas o riesgos. En segundo lugar, el análisis con relación a la propia definición de sistema político (Alcántara, 1994), en cuyo caso se darán cabida a los siguientes seis apartados: En primer lugar, definición del régimen de gobierno ideal para el país y del real existente. En segundo término, la categorización del sistema electoral existente. En tercer lugar, y con relación a los partidos políticos: número de los mismos idóneo, nivel de militancia partidista, identificación partidista, grado de actividad de los partidos, democracia interna y estructura de su partido. En cuarto lugar, y en el apartado relativo a otros actores, se tendrán en cuenta el papel y futuro de los movimientos sociales, el grado de actividad de sindicatos y empresarios en cuanto a la relación de sus demandas y la gobernabilidad, papel, futuro, evaluación y grado de actividad de las Fuerzas Armadas, así como de las iglesias. En quinto lugar se evaluará el cambio social y la definición de la sociedad en cuanto a su interacción y promoción. Por último, en el terreno de las políticas públicas, se trata de identificar problemas, evaluar la política económica: gasto público, impuestos, privatizaciones, gasto social y papel del Estado, así como la política internacional: integración, condicionalidad, procedencia del capital foráneo y prioridad de la política exterior.

Dentro del gran número de subepígrafes que recoge este apartado se pretende abordar fundamentalmente las diferencias existentes, al igual que en el apartado anterior por adscripción partidista, situación socioeconómica, valores y autoubicación ideológica en el continuo izquierdaderecha. 
3) Algunos apartados de los dos puntos anteriores, en correlación con la autoubicación ideológica en la escala numérica izquierda-derecha, permitirá abordar el grado de pertinencia del uso de dichas categorías en los países latinoamericanos con base empírica contrastándolo con las ideas ya expuestas en Alcántara (1991). Por otra parte, se pretende contrastar la distancia ideológica "referencial" entre los distintos partidos, medida gracias a la autoubicación y la ubicación del resto de los diputados y la "distancia programática" conformada por diferentes medias con relación a aspectos definitorios en torno a posiciones "de programa”. La combinación de ambos tipos de distancias permitirá establecer el potencial polarizador de los sistemas de partidos.

4) En un último apartado definido en torno a la política como profesión se analizan las motivaciones que llevan a un sujeto a emprender una carrera pública como diputado y su propia cultura política. Entre la serie de factores que impulsan a algunas personas a participar en la política, Payne et al. (1990) establecieron una tipología de incentivos entre los que se daban cabida al prestigio social, al programa, a la sociabilidad, a la obligación, a la pulsión por el juego, a la misión en cuanto a la necesidad de comprometerse con una causa trascendental que diera significado a su vida y a la adulación como necesidad exagerada de ser alabados y queridos. Algunos de estos incentivos son analizados en los siguientes siete apartados: motivaciones; problemas que son objeto de preocupación; compatibilidad con su profesión e ingresos; antecedentes familiares; experiencia y evaluación de la actividad política: años de dedicación y afiliación, continuidad partidista y autosatisfacción; funciones específicas desempeñadas; y circunscripción de elección.

Un cruce del perfil profesional del diputado con las variables relativas al género, edad, años de dedicación a la política, años de experiencia como diputado y tipo del partido al que pertenece podría satisfacer una de las hipótesis de trabajo de que los partidos nuevos o las candidaturas independientes se correlacionarían positivamente con los incentivos del juego, la misión y, posiblemente, la adulación. Paralelamente, la mayor edad y la más dilatada permanencia en la política establecerían una correlación positiva con partidos políticos consolidados y todo ello con los primeros cuatro tipos de incentivos.

Con relación a la cultura política del diputado, a efectos explicativos, puede seguirse la senda abierta por Pye (1971) en el sentido de establecer una tipología compuesta por seis formas diferentes de cultura política de la élite política. Pye las definió según los siguientes rótulos: expansiva, exclusiva, cerrada, parroquial, sintética e inexistente. Esta clasificación y la caracterización de los respectivos modelos actuará como guía taxonómica para el universo objeto del presente estudio.

En el terreno de la biografía personal se dan cabida tres grandes apartados que conformarán las principales variables dependientes de la investigación: el primero se refiere a datos biográficos primarios como son el año, lugar de nacimiento y estado civil, y a la profesión del padre y nivel de estudios de los padres; el segundo a lo que se ha venido denominando hasta aquí situación socioeconómica que da cabida a la clase social de origen, autoubicación socioeconómica, estudios y grado de asociacionismo; finalmente y en tercer lugar al ya citado apartado de valores que recoge la religiosidad, opinión sobre el aborto y el divorcio, y autoubicación ideológica.

\section{METODOLOGÍA}

La investigación propuesta se basa entonces en la medición de las actitudes, percepciones y opiniones de distintas élites parlamentarias. Mediante la utilización de este instrumento de análisis se intenta completar y superar el enfoque empleado en análisis de élites parlamentarias basados en las características socio-demográficas de los parlamentarios, especialmente utilizados en Gran Bretaña. Esto supone una ventaja considerable ya que permite adoptar una visión menos determinista del comportamiento de la clase parlamentaria, a través de la obtención de la información sobre las actitudes y opiniones de los propios parlamentarios en vez de basarse en el carácter predictivo de sus características socio-demográficas o su adscripción partidista. Diversos autores han señalado la necesidad de primar este tipo de estudio basado en la consideración de los propios parlamentarios como una de las principales fuentes de información (Crewe, 1985).

Este punto de partida implica la utilización de cuestionarios $y / o$ entrevistas en profundidad como instrumentos de análisis fundamentales del proyecto. A través de ellos se pretende no sólo obtener rasgos biográficos y socio-demográficos de los parlamentarios, con los que poder realizar tipologías de los parlamentarios, sino también sus actitudes y opiniones sobre distintos aspectos de los actores e instituciones del sistema político al que pertenecen. La utilización de cuestionarios es una de las maneras más útiles para obtener información sobre las actitudes y opiniones de una población. Evidentemente, no es la única fuente de información, pero sí la única en la que el propio diputado es el protagonista activo. Como información complementaria se pueden utilizar datos sobre el comportamiento político de los diputados, en las votaciones, discursos, etc. También se puede obtener este tipo de información mediante la realización de entrevistas en profundidad y de entrevistas semiestructuradas.

Sin embargo, en el presente estudio se ha optado por considerar el cuestionario como el primer instrumento de análisis por razones fundamentales. La primera tiene que ver con el elevado número de países que se analizan; el cuestionario, siguiendo las debidas precauciones, consigue un elevado grado de comparabilidad entre los datos obtenidos mientras que la codificación de las entrevistas en profundidad sería una tarea muy costosa, con un resultado final que posiblemente sería inferior en términos de comparabilidad de los datos. La segunda viene derivada del hecho de que la utiliza- 
ción generalizada de entrevistas en profundidad supondría un coste muy elevado tanto en tiempo como en dinero.

Dada la carencia de este tipo de datos sobre la élite parlamentaria latinoamericana, la realización de este proyecto implica la utilización de datos primarios, lo cual tiene importantes consecuencias sobre varios aspectos del trabajo. Por un lado, gran parte de los recursos materiales debieron emplearse en la recolección de información a través de cuestionarios y, en particular, en el diseño del cuestionario, ya que la fiabilidad y validez de la medición de las actitudes y opiniones depende en gran parte del buen diseño de las preguntas y de las categorías de respuesta (Alwin, 1992). Las investigaciones que emplean datos secundarios no requieren incorporar en su presupuesto ni en su planificación temporal los costes que suponen diseñar los cuestionarios, realizar las entrevistas y codificar la información. Esta inversión inicial, sin embargo, parece justificada si se piensa en las ventajas que la utilización de datos primarios tiene sobre los resultados de la información.

En primer lugar, la información se recaba siguiendo de una forma más estricta las líneas marcadas por las preguntas teóricas a las que se aspira contestar. En otras palabras, no es la no existencia de datos lo que perfila más o menos acentuadamente el contenido de la investigación, sino la propia investigación la que determina el tipo de datos que serán útiles y por lo tanto recogidos. Uno de los principales problemas de las ciencias sociales, la carencia de datos necesarios para dar respuesta a determinadas cuestiones de fundamental importancia, queda así resuelto en el presente proyecto a través de la elaboración de una base de datos original que se ajuste a las necesidades de la investigación.

En segundo lugar la recolección y explotación de datos primarios garantiza la originalidad de los resultados. Ello es aún más evidente si se tiene en cuenta la ausencia de estudios empíricos previos sobre las actitudes y características de la élite política latinoamericana.

Por último, la creación de una base de datos de estas características supone una inversión que excede los límites del presente proyecto, en la medida en que podrá ser utilizada en futuras investigaciones con otros objetivos relacionados sobre el mismo tema y en estudios de casos nacionales específicos?

En conclusión, la metodología de este trabajo responde de manera cercana a sus objetivos. Frente a una investigación que marque claramente los objetivos, hipótesis y resultados que supone va a encontrar, la utilización de los propios diputados como informantes convierte a la investigación en algo necesariamente más flexible en el que el diálogo entre conceptos y datos empíricos se intensifica.

\section{CONCLUSIÓN}

En el marco político de la América Latina contemporánea, el estudio de la clase política y de los diputados en especial adquieren una relevancia difícilmente discutible. Este estudio resulta crítico para entender las dinámicas políticas e institucionales de los países de la región. Los estudios derivados de la investigación sobre las élites políticas latinoamericanas se enmarcan precisamente en una preocupación clara por la comprensión de las especificida-

7. La metodología empleada en el proyecto concierne por lo tanto a dos aspectos fundamentales: la recogida de datos y su posterior análisis. En cuanto al primer aspecto ya se ha mencionado la elección del cuestionario como instrumento esencial de análisis. En los casos nacionales que cuentan con Asambleas Legislativas pequeñas (de número inferior a 125 diputados) se intentará que la muestra sean todos los diputados. Sin embargo, en muchos casos, la dimensión del Parlamento hace que esto no sea factible de forma que se diseñará una muestra representativa del mismo. Esta circunstancia será atinente para los casos de Argentina, Brasil, Colombia, México y Venezuela. Los criterios de estratificación en la selección de los diputados serán el partido y la procedencia regional. Los errores muestrales por países varían del $+/-4 \%$ al $+/-9 \%$ con un nivel de confianza del $95,5 \%$; el error global del estudio con el mismo margen confidencial es del $+/-2,9 \%$. Por otra parte, una consideración complementaria que debe formularse es el interés de realizar el trabajo de campo, como criterio homogeneizador, a lo largo del primer año después de la constitución del Parlamento, en un plazo, para cada caso, no superior a un mes para evitar el impacto de los cambios rápidos en la agenda, y, finalmente, por personal investigador español. Por otra parte, y habida cuenta de la profunda diferencia en la conformación de las Cámaras, el universo utilizado, en los casos de bicameralismo, se ciñe a las Cámaras Bajas.

El cuestionario se compone fundamentalmente de preguntas cerradas. Una vez más el criterio de máxima comparabilidad de los datos ha conducido a esta decisión. La utilización de preguntas cerradas (es decir, con un número limitado de categorías ya provisto al entrevistado) tiene la ventaja de que la información obtenida está estandarizada y que tienen un mayor potencial estadístico ya que al ofrecer un número limitado de respuestas posibles se evita una dispersión excesiva de la población entrevistada (Fowler, 1989). Por otra parte esto tiene la desventaja de que las respuestas proporcionadas pueden constreñir en exceso las respuestas de forma que se pierda valiosa información. En los cuestionarios empleados se ha intentado superar esta desventaja mediante la inclusión de la categoría "otros", en la que los entrevistados deben proporcionar ellos mismos la respuesta. Se ha intentado así seguir el consejo de Converse y Presser (1986) de utilizar preguntas cerradas seguidas de preguntas abiertas o de una categoría que incluya para que los individuos que no puedan elegir entre las categorías propuestas tengan opción y de esta forma no se pierda una valiosa información sobre opiniones singulares o sobre un aspecto que no ha sido previsto por los investigadores en el diseño del cuestionario.

Otras dos ventajas de las preguntas cerradas sobre las abiertas es que son más fáciles y estadísticamente más fiables de codificar. Por esta razón las preguntas cerradas suelen preferirse sobre las abiertas ya que estas últimas en ocasiones encarecen en demasía la investigación, la retardan y en ocasiones plantean problemas de comparabilidad de los datos obtenidos. Como señalan Schwarz y Hippler el formato cerrado es preferible en aquéllos casos en los que el investigador está interesado en obtener de parte de los entrevistados una evaluación completa sobre una serie de temas (Schwarz y Hippler, 1991: 44).

En la mayoría de las preguntas cerradas se han utilizado escalas de tipo Likert, en las que los entrevistados deben elegir entre varias categorías que corresponden a los diversos grados de acuerdo y desacuerdo con una afirmación leída por el entrevistador. A estas categorías se les asignan valores de forma que se puedan construir escalas con otras preguntas relacionadas dentro del cuestionario. Aunque todavía no existe consenso entre los especialistas sobre el número de categorías que proporciona el mayor grado de fiabilidad y validez parece que la discusión se centra entre la utilización de escalas de cinco o de siete categorías ya que ambas permiten medir tanto la dirección de la evaluación del entrevistado respecto a una cuestión, como la intensidad de esta opinión (Alwin, 1992). En el presente caso, se ha optado por presentar escalas de cinco categorías en aquellas preguntas que se corresponden con mostrar el grado de acuerdo o desacuerdo de los entrevistados.

Aunque el cuestionario es esencialmente el mismo para todos los países, existe la posibilidad de introducir pequeñas variaciones nacionales (que, en cualquier caso, no excederán de cinco preguntas nuevas en cada caso nacional). Por otro lado se ha tenido especial cuidado para que las preguntas signifiquen lo mismo en distintos sitios. Las entrevistas se realizarán de forma individualizada y personalizada previa cita con el entrevistado de quien se solicitará su disponibilidad a través de una carta de presentación del proyecto y del entrevistador. 
des de la representación política y de la acción de los líderes políticos en las sociedades latinoamericanas. Como tales, dichas investigaciones participan de algunos de los intereses teóricos y metodológicos que se han reseñado en distintos apartados de este artículo. Ello no significa, ciertamente, que las mismas estén guiadas por una comprensión teórica uniforme de la vida política latinoamericana. Pero sí que apunta la existencia de unos campos de investigación, y de unas preguntas teóricas y empíricas cuya dilucidación pueden resultar de sumo interés para el conocimiento de la vida política latinoamericana.

Al mismo tiempo, estos estudios participan de la unidad y coherencia que proporciona la información derivada de las encuestas a los diputados latinoamericanos. Este común denominador, de carácter metodológico, ha permitido a diversos investigadores abordar problemas fundamentales de la vida política latinoamericana de una forma sistemática, comparable, estadísticamente discernible. A nuestro juicio, la metodología homogénea de estos estudios y el impulso de comprensión común que subyace a cada uno de los trabajos que han resultado de estos análisis indican que la misma se inscribe en una agenda de investigación que es ambiciosa y que, al mismo tiempo, está sólidamente fundamentada.

\section{BIBLIOGRAFÍA CITADA}

AberbaCH, Joel D., Robert D. Putnam y Bert A. Rockman (1981). Bureaucrats and Politicians in Western Democracies. Cambridge University Press. Cambridge.

Aguero Piwonka, Felipe y Mariano Torcal Loriente (1993). "Elites, factores estructurales y democratización (una discusión de aportes recientes en la literatura)". Revista de Estudios Políticos (Nueva Época). Núm. 80 Abril-Junio, págs. 329-350.

AlCÁNTARA, Manuel (1989 y 1990). Sistemas políticos de América Latina. Tecnos. Madrid. 2 vols.

- (1991). "La relación izquierda-derecha en la política latinoamericana". Leviatán. Madrid.

- (1993). "Cuando hablamos de ciencia política, ¿de qué hablamos?. Revista Mexicana de Sociología. 4193. Págs. 147-178.

- (1994). Gobernabilidad. crisis y cambio. Centro de Estudios Constitucionales. Madrid.

AlWIN, D. F. (1992). "Information Transmition in the Survey Interview: Number of Response Categories and the Reliability of Meausuremet" en P. V. Marsden (ed.) Social Methodology. Basil Blackwell. Oxford.

ARENDT, Hannah (1993). Was ist Politik? Piper. Munich y Zurich.

BEST, Heinrich (1995). "European Parliamentary Elites: Cleavage Structures, Conflict Patterns and Party Systems". Ponencia presentada en la XIII sesión Anual del ECPR, 27 de abril-2 de mayo, Burdeos.
Beyme, Klaus von (1995). La clase política en el estado de partidos. Alianza. Madrid.

BorCherT, Jens y Lutz Golsch (1995). "Die Politische Klasse in Westlichen Demokratien: Rekrutierung, Karriereinteressen un institutioneller Wandel". Politische Verteljaresschrift, 36, Jng., Heft 4, 5, Diciembre.

BORGATTI, et al (1992). UNICET IV Network Analysis Software.

Bourdieu, Pierre (1990). Language and Sumbolic Power. Harvard University Press. Cambridge.

Bravo Ahuja, Marcela (1986). "La élite política en México". Revista Mexicana de Ciencias Políticas y Sociales. México. Julio-Septiembre. Págs. 71-93

BRESSER PEREIRA, Luiz Carlos, José María Maravall y Adam Przeworski (1993). Economic Reforms in New Democracies. Cambridge University Press. Cambridge.

Cain, Bruce E., John A. Ferejohn y Morris P. Fiorina (1979). "The House is not a Home: British MPs in their Constituencies". Legislative Studies Quarterly. Vol. 4, n 4 , noviembre, págs. 501-523.

Cano, J. y Antonio Porras (ed.) (1994). Parlamento y consolidación democrática.

Close, David (ed.) (1995). Legislatures and the New Democracies in Latin America. Lynne Rienner Pub. Boulder.

Collier, David (1991). "The Comparative Method: Two Decades of Change" en Rustow, D. y Ericsson, K. (ed.) Comparative Political Dynamics: Global Research Perspectives. Free Press. New York.

Converse, J. y S. Presser (1986). Survey Questions: Handcrafting the Standarized Questionnaire. Sage Manuscript 63. Beverly Hills.

CotARELo, Ramón (1990). En torno a la teoría de la democracia. Centro de Estudios Constitucionales. Madrid.

CRESPO, Ismael (1995). "Valores democráticos e identificación ideológica de los parlamentarios centroamericanos". Huarte de San Juan. Revista de la Facultad de Ciencias Humanas y Sociales. $\mathrm{N}^{\circ}$ 2, págs. 159-184.

CREWE, Ivor (1985). Electoral change in Western democracies: Patterns and sources of electoral volatility. St. Martin Press. New York.

DEXTER, L. A. (1970). Elite Specializeed Interviewing. Northwestern University Press. Evanston.

DunN, John (1996). The History of Political Theory. Cambridge University Press. Cambridge.

Eliassen, Kjell A. (1995). The Professionalization of the Elite Recruitment. Ponencia presentada en la Xlll Sesión Anual del ECPR, 27 de abril-2 de mayo, Burdeos.

Evans, Mark (1995). "Elitism", en David Marsh y Gerry STOKER (eds.): Theory and Methods in Political Science. MacMillan. Londres. 
FOWLER, F. J. (1988). Survey Research Methods. Sage. Newbury Park.

GuNTHER, Richard (ed ) (1992). Elites and democratic consolidation in Latin America and Southern Europe. Cambridge University Press. Cambridge.

Henerson, M. E., L.L. Morris, y C.T Fitz-Gibbon,. (1987). How to meausure attitudes. Sage. Newbury Park.

KING, Anthony (1981). "The Rise of the Career Politician in Britain and Its Consequences", en British Journal of Political Science, vol.11, núm. 3, págs. 249-286.

LAMBERT, Jacques (1973). América Latina. Ariel. Barcelona.

LIJPHART, Arend (1971). "Comparative politics and comparative method". American Political Science Review 65, 683-693.

LINZ, Juan J. et al. (1990). Hacia una democracia moderna. La opción parlamentaria. Ediciones Universidad Católica de Chile.

- (1992). Los problemas de las democracias y la diversidad de las democracias. Universidad Autónoma de Madrid.

LinZ, Juan J. y Arturo Valenzuela (1994). The Failure of Presidential Democracy. 2 vol. The Johns Hopkins University Press. Baltimore.

LiPSET, Seymour M. 1967. "Values, Education, and Entrepreneurship", en Seymour M. Lipset y Aldo Solari. Elites in Latin America. Nueva York. Oxford University Press.

LIPSET, Seymour M. y Aldo Solari, (ed.) (1967). Elites in Latin America. Oxford University Press. New York.

Lujambio, Alonso (1993). "Presidentes y Congresos: Estados Unidos, la experiencia latinoamericana y el futuro mexicano". Foro Internacional. XXXI 11-3, págs. 517-560.

MarCH, James, G. y Johan P. Olsen (1984). "The New Institutionalism: Organizational Factors in Political Life". The American Political Science Review. 78.3. Págs. 734-749.

MarTZ, John D. (1992). "Party Elites and Leadership in Colombia and Venezuela". Journal of Latin American Studies. Cambridge University Press. 24.1. Págs. 87121.

Matthews, Donald (1960). U.S. Senators and TheirWorld. Random House. Nueva York.

- (1985). "Legislative Recruitment and Legislative Careers", en Gerhard LoEwenkeG, Samuel Patterson y Malcolm E. Jewell (edits.): Handbook of Legislative Research (Cambridge: Harvard University Press).

Molinelli, Guillermo (1991). Presidentes y Congresos en Argentina: mitos $y$ realidades. Grupo Editor Latinoamericano. Buenos Aires.

Moser, C. y G. Kalton (1983). "Scaling Methods" en Survey Methods in Social Investigation. Págs. 350-377. Gower. Aldershot.
OAKESHOTT, Michael (1991). Rationalism in politics and other essays. Liberty Press. Indianapolis.

O'DONNELL, Guillermo. 1996. "Otra institucionalización". Ágora. Invierno, n 5 , págs. 5-28.

Payne, James L.; Oliver H. Woshinsky; Eric P. Veblen; William H. Coogan; y Gene E. Bigles (1990). Las motivaciones de los politicos. Los factores que impulsan a algunas personas a participar en la política. Editorial Limusa. México.

PyE, Lucien W. (1971). "Identity and the Political Culture". En Binder et al Crises and Sequences in Political Development. Princeton University Press. Princeton.

PIORE, Michael J. (1995). Beyond Individualism. Harvard University Press. Cambridge y Londres.

Putnam, Robert D. (1973). The beliefs of Politicians. Ideology. Conflict and Democracy in Britain and Italy. Yale University Press. New Haven.

Ramos, María Luisa (1997). "Creencias y valores de los parlamentarios en Venezuela". Nueva Sociedad. $\mathrm{N}^{\circ}$ 148, marzo-abril, págs. 44-50.

Requejo, Ferrán (1990). Las democracias. Democracia antigua. Democracia liberal y Estado de Bienestar. Ariel. Barcelona.

ROHRSCHNEIDER, Robert (1994). "Report from the laboratory: The influence of institutions on political elites' democratic values in Germany". American Political Science Review. Vol. $88 \mathrm{n}^{\circ}$ 4. 927-941.

RokKan, S.; S. Verba; J. Viet, y E. Almasy (1969). Comparative Survey Analysis. Mouton. The Hague.

RuOSTETSAARI, Ilkka (1995). "Transformation of the Finnish Parliamentary Elites in the 20th Century". Ponencia presentada en la Xlll sesión anual del ECPR, 27 de abril-2 de mayo, Burdeos.

RuESCHEMEYER, Dietrich, Evelyne Huber Stephens y John D. Stephens (1992). Capitolist Development and Democracy. The University of Chicago Press. Chicago.

Santamaría, Julián (1994). "El papel del Parlamento durante la consolidación de la democracia y después" Revista de Estudios Políticos. Madrid. Abril-Junio 9-26.

SARTORI, Giovanni (1988). Teoría de la democracia. Alianza. Madrid.

Schmitt, Carl (1982). Teoria de la Constitución. Alianza Universidad. Madrid.

SCHWARZ, N. y H. Hippler (1991). "Response Alternatives: the Impact of their Choice and Presentation Order" en Biemer, et al. (eds.) Measurement Error in Surveys. John Wiley \& Sons.

SeAring, Donald D. (1994). Westminster's World: Understanding Political Roles. Cambridge y Londres.

SMELSER, Neil J. (1976). Comparative Methods in the Social Sciences. Prentice Hall. Englewood Cliffs. 
Suleiman, Ezra y Henri Mendras (eds.) (1995). Le recrutement des élites en Europe. Éditions La Découverte. Paris.

TheLEN, Kathleen y Sven Steinmo (1992). "Institutionalism in comparative politics", en Sven Steinmo, Kathleen Thelen y Frank Longstreth (eds): Structuring politics: Historical institutionalism in comparative analysis. Cambridge University Press. Nueva York.

WAHLKE, John, Heinz Eulau; William Buchanan y Leroy Ferguson, 1962. The Legislative System. D.C. Head and Co. Lexington.
WeIsBERG, H. F. y B.D. Bowen (1977). An Introduction to Survey Research and Data Analysis. Freeman. San Francisco .

Westholm, Anders y Richard G. Niemi. (1992). "Political Institutions and Political Socialization. A CrossNational Study". Comparative Politics. 25.1. Octubre. Págs. 25-41.

WiARDA, Howard J. (1981). Corporatism and National Development in Latin America. Westview Press. Boulder:

\section{RESUMEN}

El presente artículo describe algunas de las características básicas del proyecto de investigación sobre los diputados latinoamericanos realizado por el equipo de investigación sobre élites políticas latinoamericanas de la Universidad de Salamanca. Además, este artículo señala algunas de las razones fundamentales por las que este tipo de investigación es relevante en los terrenos académico y político. Finalmente, este artículo define y clasifica las principales alternativas teóricas presentes en el estudio de las élites políticas, así como apunta algunas de las expectativas e hipótesis que se derivan de cada una de ellas.

\section{ABSTRACT}

This article defines some basic characteristics of the research project on Latin American members of parliament conducted by the University of Salamanca research team on Latin American political elites. In addition, it points out some of the reasons why this kind of research is both scholarly and politically interesting. Finally, this article defines and classifies the main alternative theoretical approaches to the study of political elites, as well as some of the empirical expectations that derive from each theoretical framework. 